\section{Ein organisches Molekül mit einem starren, nanometergroßen Hohlraum}

Peter Timmerman, Willem Verboom, Frank C. J. M. van Veggel, Willem P. van Hoorn und David N. Reinhoudt*

Das sich schnell entwickelnde Gebiet der supramolekularen Chemie ${ }^{[1-3]}$ hat eine Vielzahl nützlicher Bausteine zur Synthese von künstlichen Rezeptormolekülen wie Calixarene ${ }^{[4]}$, Cyclodextrine $^{[5]}$ und Cavitanden auf Resorcin-Basis ${ }^{[6]}$ hervorgebracht. Die fast unendliche Zahl von molekularen Rezeptoren in der Natur entsteht durch systematische Kombination einer begrenzten Zahl von Struktureinheiten. Wir versuchen zur Zeit, durch Verknüpfen von einfachen starren Bausteinen Wirtmoleküle auf analoge Weise herzustellen ${ }^{[7]}$. Vor kurzem konnten so neue Rezeptormoleküle mit einzigartigen Komplexierungseigenschaften synthetisiert werden ${ }^{[8-11]}$, die kleine Hohlräume, in denen Alkalimetall-Kationen, Anionen und kleine organische Moleküle koordiniert werden können, aufweisen. Bei der Synthese von Rezeptormolekülen mit großen Hohlräumen ist generell problematisch, daß solche Hohlräume wegen der Flexibilität großer organischer Moleküle in sich zusammenbrechen. Kürzlich stellten Sanders et al. ${ }^{[12]}$ Rezeptormoleküle mit großen, relativ offenen, starren Hohlräumen her, indem sie starre, planare Komponenten (Porphyrine und Derivate der Cholsäure) über kovalente Einfachbindungen verknüpften.

Wir beschreiben hier einen neuen Zugang zu Rezeptormolekülen mit großen Hohlräumen, die aus Bausteinen, die selbst einen Hohlraum aufweisen, nämlich Calix[4]arene und Cavitanden mit Resorcin-Grundgerüst, aufgebaut werden. Die kreisförmige Anordnung von vier solchen Untereinheiten führt mithilfe eines konvergenten Synthesewegs zur Bildung des Holanden $\mathbf{1}^{[13]}$. Dieser ist ein extrem starres Wirtmolekül, in dem die Hohlräume der vier Komponenten zu einem abgeschirmten, nanometergroßen Hohlraum verschmelzen.
Als Teil unserer Untersuchungen zur Synthese von selektiv funktionalisierten Resorcin-Cavitanden ${ }^{[14]}$ haben wir die Reaktion von Cavitand $2^{[15]}$ mit dem am oberen Rand 1,2-funktionalisierten Calix[4]aren 3 untersucht. Verbindung 3 wurde in einer Gesamtausbeute von $72 \%$ durch Reduktion der entsprechenden 1,2-Dinitroverbindung ${ }^{[16]}$ und anschließende Reaktion mit zwei Äquivalenten $\alpha$-Chloracetylchlorid hergestellt. Setzt man 2 mit 3 im Verhältnis $1: 1$ in $\mathrm{CH}_{3} \mathrm{CN} / \mathrm{Cs}_{2} \mathrm{CO}_{3} / \mathrm{KI}$ um, so entsteht in $20 \%$ Ausbeute $\mathbf{4} \mathbf{a}$, bei der die Calix[4]aren-Einheit in 1,2-Stellung, d. h. an zwei benachbarte Arenringe von 2 mit endo-Stereochemie gebunden ist, und $32 \%$ des exo-Isomers $4 b^{[17]}$. Neben diesen 1:1-Addukten entstanden geringe Mengen der drei möglichen isomeren 2:1-Produkte 5a-c. Verbindungen, in denen die Calix[4]aren-Einheit in 1,3-Stellung, d.h. an gegenüberliegende Arenringe des Cavitanden 2 gebunden ist, konnten nicht nachgewiesen werden. Bei der Reaktion von 2 mit zwei Äquivalenten 3 wurden nur die 2:1-Additionsprodukte in nahezu statistischem Verhältnis von endo-endo- (5a, abgebildet), endo-exo- (5b) und exo-exo-Produkt (5c) in einer Gesamtausbeute von $64 \%$ isoliert $^{[18]}$.

Die Bildung des exo-1:1-Produkts ist bei dieser Reaktion leicht bevorzugt. Das endo-1:1-Produkt entsteht in größeren Anteilen, wenn funktionelle Gruppen am Calix[4]aren eingeführt werden, die den entsprechenden Übergangszustand durch Wechselwirkungen mit dem Cavitanden begünstigen. Die Reaktion eines 1:1-Gemischs von 2 mit dem Calix[4]aren $6^{[19]}$, das zwei zusätzliche Nitrogruppen enthält, führte ausschlieBlich zum endo-1:1-Isomer 7 und kleinen Mengen der 2:1-Produkte $8 \mathbf{a}$ (endo-endo) und $\mathbf{8 b}$ (endo-exo). Da 7 instabil ist, wurden seine freien Hydroxygruppen silyliert ${ }^{[20]}$, und das Produkt 9 in $41 \%$ Ausbeute isoliert. Die Nitrogruppen in 9 ließen sich mit Raney-Ni/Hydrazin ${ }^{[16]}$ leicht zu Aminogruppen reduzieren. Die anschließende Umsetzung mit $\alpha$-Chloracetylchlorid lieferte das Bis(2-chloracetamid)-Derivat 10 quantitativ. Dies wurde in Dimethylformamid (DMF; $5 \mathrm{~mm}$ ) gelöst und mit CsF bei $80^{\circ} \mathrm{C}$ desilyliert; die Lösung wurde danach 48 Stunden in Gegenwart von $\mathrm{Cs}_{2} \mathrm{CO}_{3}$ und $\mathrm{KI}$ gerührt, wobei zwei Reaktionsprodukte entstanden, von denen das polarere ( $27 \%$ isolierte Ausbeute) der aus zwei Calix[4]aren-Einheiten bestehende Carcerand 11 ist, in dessen Inneren ein DMF-Molekül eingeschlossen ist. Das Vorhandensein dieses Gastmoleküls wurde sowohl durch FAB-Massenspektrometrie $(m / z=2126(100 \%)[M+\mathrm{DMF}+$ $\left.\left.\mathrm{Na}^{+}\right]\right)$als auch durch ${ }^{1} \mathrm{H}$-NMR-Spektroskopie nachgewiesen (die zwei Methylgruppen des DMF-Moleküls ergeben zwei Singuletts bei $\delta=0.66$ und $\left.-0.86^{[21]}\right)$.

Das zweite Produkt (26\% isolierte Ausbeute) war das Zielmolekül, der Holand 1. Diese Verbindung konnte auch durch tropfenweise Zugabe einer äquimolaren Lösung von $12^{[22]}$ und 2 in DMF zu einer Suspension von $\mathrm{Cs}_{2} \mathrm{CO}_{3}$ und $\mathrm{KI}$ in DMF in $35 \%$ Ausbeute erhalten werden. Das FAB-Massenspektrum belegt die Bildung der 2:2-Struktur $(m / z=4084 \quad(100 \%)$ $\left.\left[M+\mathrm{Na}^{+}\right]\right)$, und das linienarme ${ }^{1} \mathrm{H}-$ NMR-Spektrum (Abb. 1) spiegelt den hohen Symmetriegrad, der für 1 zu erwarten ist, wider. Für seine Größe ist 1 extrem starr: Die Calix[4]aren- und Cavitandeinheiten, die selbst starr sind, werden über zwei unbewegliche Spacer miteinander

[*] Prof. Dr. Ir. D. N. Reinhoudt, Dr. P. Timmerman, Dr. W. Verboom,

Dr. Ir. F. C. J. M, van Veggel, Ir. W. P. van Hoorn

Laboratory of Organic Chemistry, University of Twente

P.O. Box 217, NL-7500 AE Enschede (Niederlande)

Telefax: Int. $+53 / 356024$ 


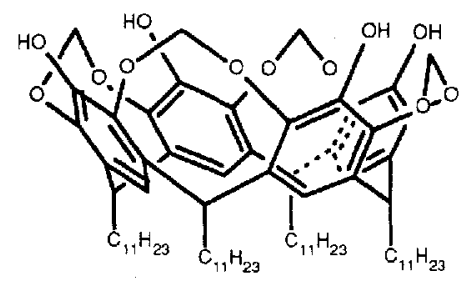

2

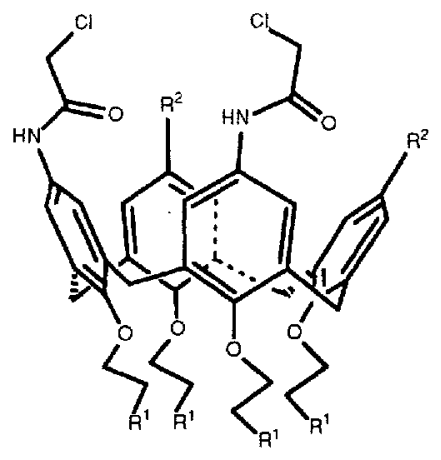

3: $\mathrm{R}^{1}=\mathrm{OCH}_{2} \mathrm{CH}_{3}, \mathrm{R}^{2}=\mathrm{H}$

6: $\mathrm{R}^{1}=\mathrm{CH}_{3}, \mathrm{R}^{2}=\mathrm{NO}_{2}$

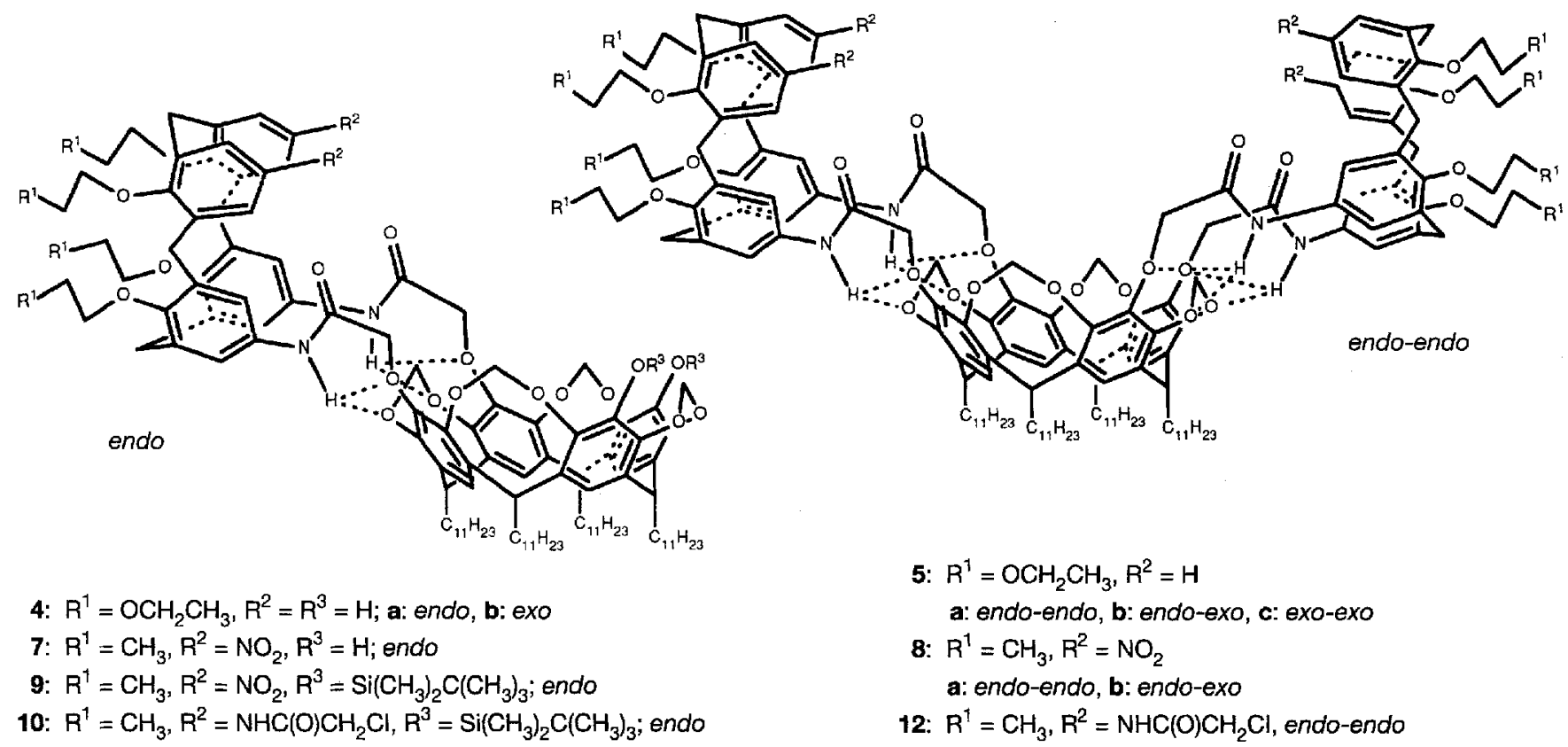

verbunden. Dies wird beim Vergleich der ${ }^{1} \mathrm{H}-\mathrm{NMR}$-Spektren der Verbindungen 3, 5a und $\mathbf{1}$ deutlich. In $\mathbf{3}$ unterscheiden sich die Signale der aromatischen Protonen in ortho-Stellung zur Amidfunktion aufgrund der nahezu ungehindert möglichen Rotation um die C(Aren)-N-
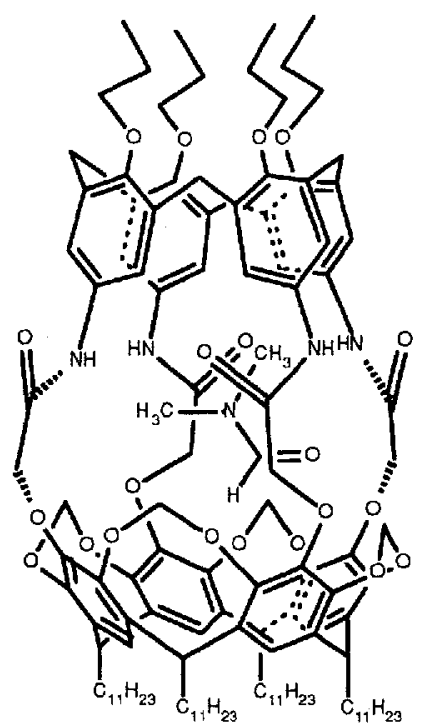

11
Bindung in ihrer chemischen Verschiebung nur um $0.08 \mathrm{ppm}$. In $\mathbf{5} \mathbf{a}$ ist der $\Delta \delta$-Wert viel größer, da zwei dieser Calixareneinheiten mit dem Cavitanden verknüpft sind. Die Differenz von $0.85 \mathrm{ppm}$ für $\mathbf{5} \mathbf{a}$ ist ein Zeichen für die geringe Flexibilität der Amidspacer in dieser Verbindung. Im Holanden 1 ist die Beweglichkeit der Calixarenbausteine noch stärker eingeschränkt, woraus ein $\Delta \delta$-Wert von 1.0 resultiert. Die Starrheit der Struktur von 1 wird durch einen Vergleich der chemischen Verschiebungen der zwei Methylenprotonen im Spacer von 3, 5a und 1 noch deutlicher. Während diese
Protonen in 3 ein Singulett ergeben, spaltet dieses in $\mathbf{5}$ a zu einem AB-System mit $\Delta \delta=0.4$ auf. Die Bewegung der Calixareneinheiten durch Rotation um die $\mathrm{O}-\mathrm{CH}_{2}$-Bindung im Spacer ist in

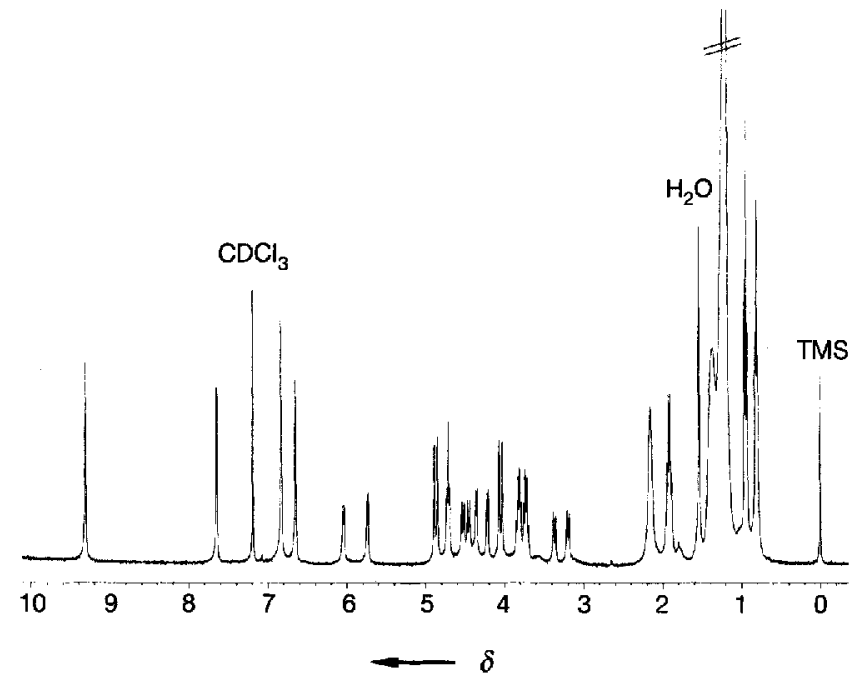

Abb. 1. ${ }^{1} \mathrm{H}-\mathrm{NMR}-\mathrm{Spektrum}(400 \mathrm{MHz})$ von 1 in $\mathrm{CDCl}_{3}$ bei Raumtemperatur. 
5 a aber weiterhin möglich. In 1 ist auch diese Rotation vollständig eingefroren, und der $\Lambda \delta$-Wert zwischen den beiden AB-Dubletts beträgt 0.85 . Die Starrheit von 1 beruht zum Teil auf den Dreizentren-Wasserstoff-Bindungen der Amid-Wasserstoffatome mit den Sauerstoffatomen im Spacer einerseits und den Sauerstoffatomen der Methylendioxybrücke des Cavitanden andererseits. Diese Bindungen sind in 1 durch gestrichelte Linien gekennzeichnet $^{[23]}$.

Wenn Verbindung 10 höherkonzentriert (11 mM) eingesetzt wurde, sanken die Ausbeuten von 27 (11) und $26 \%$ (1) auf $12 \%$ (11 und 1) ab. Offensichtlich dominiert die Polymerisation bei dieser Konzentration.

Der Hohlraum in 1 ist gemäß CPK-Modellen etwa 1.5 und $2.0 \mathrm{~nm}$ lang (Achsenlänge), und das berechnete Innenvolumen beträgt ungefähr $1.0 \mathrm{~nm}^{3}\left(1000 \AA^{3}\right)$. Abbildung 2 zeigt die Ener-

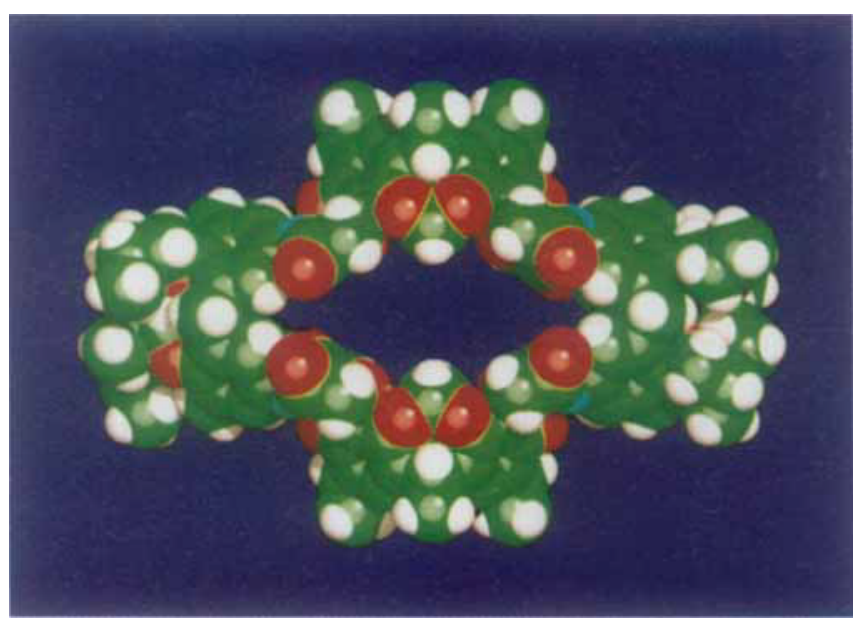

Abb. 2. Energie-minimierte Struktur des Holanden 1 (die Undecylketten sind zur Vereinfachung gegen Methylgruppen ausgetauscht worden).

gie-minimierte Struktur von 1. Die Dynamik dieser Struktur in einem $\mathrm{CHCl}_{3}$-Käfig der Kantenlänge $46 \AA$ wurde simuliert $(50 \mathrm{ps}, 300 \mathrm{~K})$. Während dieser Simulation lagerten sich vier Chloroform-Moleküle in den Hohlraum ein, ohne diesen wieder zu verlassen oder dessen Form zu verändern. Dies belegt ebenfalls die starre Struktur von 1. Die Anordnung der Amidspacer konnte während der Simulation genau verfolgt werden: Obwohl die Wasserstoff-Brückenbindungen manchmal brachen, wurden sie nach kurzer Zeit neu gebildet: Eine Rotation um die C(Aren)-NBindung wurde nicht beobachtet.

Der Holand 1 sollte einzigartige Komplexierungseigenschaften aufweisen. Die Größe des Hohlraumes ermöglicht die Komplexierung von Wirtmolekülen, die selbst gute Komplexierungsreagentien sind. Solche Reaktionen werden zur Zeit untersucht.

Eingegangen am 28. Dezember 1993 [Z 6587]

[1] J. M. Lehn, Angew. Chem. 1990, 102,1347-1362; Angew. Chem. Int. Ed. Engl. $1990,29,1304-1319$.

[2] C. J. Pedersen in Synthetic Multidentate Macrocyclic Compounds (Hrsg.: R. M. Izatt, J. J. Christensen), Academic Press, New York, 1978.

[3] a) D. J. Cram, K. N. Trueblood, Top. Curr. Chem. 1981, 98, 43-106; b) K. N. Trueblood, C. B. Knobler, E. F. Maverick, R. C. Helgeson, S. B. Brown, D. J. Cram, J. Am. Chem. Soc. 1981, 103, 5594-5596.

[4] a) C. D. Gutsche in Calixarenes, Monographs in Supramolecular Chemistry, Vol. 1 (Hrsg.: J. F. Stoddart), The Royal Society of Chemistry, Cambridge, 1989; b) Calixarenes: a Versatile Class of Macrocyclic Compounds (Hrsg.: J. Vicens, V. Böhmer), Kluwer, Dordrecht, 1991.
[5] J. Szejtli in Cyclodextrin Technology (Hrsg.: J. Szejtli), Kluwer, Dordrecht, 1988.

[6] a) D. J. Cram, S. Karbach, H.-E. Kim, C. B. Knobler, E. F. Maverick, J. L. Ericson, R. C. Helgeson, J. Am. Chem. Soc. 1988, 110, 2229 2237; b) J. A. Tucker, C. B. Knobler, K. N. Trueblood, D. J. Cram, ibid. 1989, 11t, $3688-$ 3699.

[7] L. C. Groenen, D. N. Reinhoudt, Calix/4]aremes, Molecular Platforms for Supramolecular Structures in Supramolecular Chemistry (Hrsg.: V. Balzani, L. De Cola), Kluwer, Dordrecht, 1992, S. 51.

[8] a) E. Ghidini, F. Ugozzoli, R. Ungaro, S. Harkema, A. A. El-Fadl, D. N. Reinhoudt, I. Am. Chem. Soc. 1990, 112, 6979-6985; b) E. J. R. Sudhölter, P. D. Van der Wal, M. Skowronska-Ptasinska, A. Van den Berg, P. Bergveld, D. N. Reinhoudt, Recl. Trav. Chim. Pays-Bas 1990, 109, 222-225; c) Z. Brzozka, B. H. M. Lammerink, D. N. Reinhoudt, E. Ghidini, R. Ungaro, J. Chem. Soc. Perkin Trans. 2 1993, 1037-1040; d) W. F. Nijenhuis, E. G. Buitenhuis, F. De Jong, E. J. R. Sudhölter, D. N. Reinhoudt, J. Am. Chem. Soc. 1991, $113,7963-7968$.

[9] W. I. Iwema Bakker, M. Haas, C. Khoo-Beattie, R. Ostaszewski, S. M. Franken. H. J. den Hertog, Jr., W. Verboom, D. de Zeeuw, S. Harkema, D. N. Reinhoudt, J. Am. Chem. Soc. 1994, 116, 123-133.

[10] W. F. Van Straaten-Nijenhuis, A. R. van Doorn, A. M. Reichwein, F. de Jong, D. N. Reinhoudt, J. Am. Chem. Soc. 1991, 113, 3607-3608.

[11] D. M. Rudkevich, Z. Brzozka, M. Palys, H. Visser, W. Verboom, D. N. Reinhoudt, Angew. Chem. 1994, 106, 480-482; Angew. Chem. Int. Ed. Engl. 1994, $33,467-468$.

[12] a) S. Anderson, H. L. Anderson, J. K. M. Sanders, Acc. Chem. Res. 1993, 26, $469-475$; b) L. G. Mackay, R. P. Bonar-Law, J. K. M. Sanders, J. Chem. Soc. Perkin Trans. 1 1993, 1377-1378; c) R. P. Bonar-Law, L. G. Mackay, J. K. M. Sanders, J. Chem. Soc. Chem. Commun. 1993, 456-458.

[13] Ein Holand ist ein Ligand mit einem permanenten Hohlraum.

[14] P. Timmerman, M. G. A. van Mook, W. Verboom, G. J. van Hummel, S. Harkema, D. N. Reinhoudt, Tetrahedron Lett. 1992, 33, 3377-3380.

[15] Die Herstellung dieser Verbindung erfolgte gemäß: J. C. Sherman, C. B. Knobler, D. J. Cram, J. Am. Chem. Soc. 1991, 113, 2194 - 2204.

[16] J.-D. van Loon, J. F. Heida, W. Verboom, D. N. Reinhoudt, Recl. Trav. Chim. Pays-Bas 1992, 111, 353-359.

[17] Die Konfiguration wurde mit Hilfe von NOE-Experimenten bestimmt. In einer $\mathbf{5 c}$ sehr ähnlichen Verbindung, bei der die Undecylketten gegen Methylgruppen ausgetauscht worden waren, wurde ein geringer Abstand zwischen den Protonen der unsubstituierten Arene und denen der Methylgruppen beobachtet. Dies deutet auf die exo-Konfiguration dieser Verbindung hin. Die Konfigurationen aller anderen Verbindungen konnten durch Vergleich ihrer ${ }^{1} \mathrm{H}-\mathrm{NMR}$ Spektren mit dem von $5 \mathrm{c}$ auf einfache Weise bestimmt werden.

[18] Die Verbindungen 5a-c haben große hydrophobe Oberflächen und komplexieren in $\mathrm{CDCl}_{3}$ selektiv einige Corticosteroide. Diese Ergebnisse werden an anderer Stelle veröffentlicht.

[19] P. Timmerman, W. Verboom, D. N. Reinhoudt, A. Arduini, S. Grandi, A. R. Sicuri, A. Pochini, R. Ungaro, Synthesis 1994, 185-189.

[20] Das Reaktionsgemisch wurde aufgrund der Instabilität von 7 gegenüber Kięselgel ohne Reinigungsschritt zunächst mit 20 Äquiv. tert-Butyldimethylsilylchlorid, 20 Äquiv. $\mathrm{NEt}_{3}$ und katalytischen Mengen an Dimethylaminopyridin in $\mathrm{CH}_{2} \mathrm{Cl}_{2} 24 \mathrm{~h}$ lang bei Raumtemperatur silyliert.

[21] Dieser EinschluB von Lösungsmittelmolekülen wurde früher schon oft von Cram beobachtet, siehe Lit. [15] und a) D. J. Cram, S. Karbach, Y. H. Kim, L. Baczynskyi, K. Marti, R. M. Sampson, G. W. Kalleymeyn, J. Am. Chem. Soc. 1988, 110,2554-2560; b) J. C. Sherman, D. J. Cram, ibid. 1989, 111, 45274528; c) J. A. Bryant, M. T. Blanda, M. Vincenti, D. J. Cram, J. Chem. Soc. Chem. Commun. 1990, 1403-1405. Die Stabilität von 7 wurde durch ein Austausch-Experiment in [ $\left.\mathrm{D}_{7}\right] \mathrm{DMF}$ bei $100^{\circ} \mathrm{C}(1 \mathrm{~h})$ bestimmt. Bei dieser Temperatur wurde kein DMF-Austausch beobachtet. Weitere Untersuchungen an diesen Carceranden werden noch veröffentlicht.

[22] Verbindung 12 wurde, in gleicher Weise wie 10 aus 9 , quantitativ aus 8 a hergestellt.

[23] Hinweise auf diese Wasserstoff-Brückenbindungen wurden durch quantitative NOE-Analysen von $\mathbf{5}$ a erhalten, bei der der Abstand zwischen den aromatischen Protonen in ortho-Stellung zur Amidfunktion und dem äußeren Methylenproton $\mathrm{zu} 2.5 \AA$ und der Abstand zwischen den Amidprotonen und dem äußeren Methylenproton zu $2.8 \AA$ bestimmt wurde. 